\title{
Antigenic analysis of Clostridium chauvoei flagella with protective and non-protective monoclonal antibodies
}

\author{
Yutaka Tamura, ${ }^{*}$ Mayumi KiJima, Khoji Ohishi, Toshio Takahashi, Shoko SuzUki and \\ MASAYUKI NAKAMURA
}

National Veterinary Assay Laboratory, 1-15-1 Tokura, Kokubunji, Tokyo 185, Japan

(Received 12 July 1991; revised 5 November 1991; accepted 18 November 1991)

\begin{abstract}
Five monoclonal antibodies (mAbs) directed against the flagellin of Clostridium chauvoei were used to analyse the structural and antigenic characteristics on the bacterial flagellar surface. Immune electron microscopy showed that three protective mAbs recognized the surfaced-exposed epitopes on the flagellar filament of this bacteria. In contrast, two non-protective mAbs recognized internal epitopes of the flagellar filament. These findings have been confirmed by ELISA using mAbs absorbed with whole cells of $C$. chauvoei possessing flagella. Competitive binding assays showed that protective mAbs indicated reciprocal competition, while each of the non-protective mAbs had topographically distinct epitopes. Moreover, immunoblotting analysis with cyanogen-bromide-cleaved flagellin showed that protective mAbs may preferentially recognize conformational epitopes, whilst one of the nonprotective mAbs may recognize a linear and conformation-independent epitope in the flagellin of $C$. chauvoei.
\end{abstract}

\section{Introduction}

Many studies on the flagella of Vibrio cholerae (Attridge \& Rowly, 1983), Salmonella typhimurium (Carsiotis et al., 1984; Weinstein et al., 1984), Pseudomonas aeruginosa (Montie et al., 1982) and Campylobacter jejuni (Morooka et al., 1985; Newell et al., 1985) suggest that flagella may be important as a virulence factor in the pathogenesis of infections caused by these organisms. Further studies also suggest that flagella of $V$. cholerae (Yancey et al., 1979; Resnick et al., 1980) and $P$. aeruginosa (Montie et al., 1987) may elicit production of protective antibodies against the respective infections.

Our previous studies have indicated that in Clostridium chauvoei, which causes blackleg in cattle, sheep and other ruminants, the flagella are important for protective immunity in mice. Purified flagella of $C$. chauvoei showed a protective effect, while the protective antigenicity of non-flagellated mutants was 100 -fold less than that of the flagellated parent strain (Tamura et al., 1984; Tamura \& Tanaka, 1984). However, there are still many points to be clarified as to the nature of protective antigens on the flagella of $C$. chauvoei. Recently, we obtained five monoclonal antibodies (mAbs) directed to

\footnotetext{
- Author for correspondence. Tel. 42321 1841; fax 423211769.

Abbreviations: HRPO, horseradish peroxidase; mAb, monoclonal antibody.
}

the flagella of $C$. chauvoei (Tanaka et al., 1987). Among them, three had a protective effect on mice, but the remaining $\mathrm{mAbs}$ did not, even though all reacted with the $56 \mathrm{kDa}$ protein, which corresponds to flagellin.

In the present study, we have analysed the structural and antigenic characteristics of the protective and nonprotective antigen on the flagella of $C$. chauvoei using these mAbs.

\section{Methods}

$m A b s$. Hybridomas producing $m A$ bs against the flagellin of $C$. chauvoei were prepared from splenocytes of BALB/c mice immunized with purified flagella of $C$. chauvoei strain Okinawa and murine myeloma cells (P3-X63-Ag8-U1) as described previously (Tanaka et al., 1987). Hybridoma cells were injected into the peritoneal cavity of pristane-treated BALB/c mice; ascites were used as $\mathrm{mAbs}$. The $\mathrm{mAbs}$ used in this study are listed in Table 1 . Three mAbs, Mo-41, Mo-62 and Mo-90, showed passive protective effects $(90,50$ and $50 \%$, respectively), but the other two, Mo-114 and Mo-2-60, did not (Tanaka et al., 1987).

For the absorption test, mAbs were absorbed with formalin-treated whole cells of $C$. chauvoei strain Okinawa. Wet cells in the late exponential growth phase were added to the mAb solutions at a concentration of $100 \mathrm{mg} \mathrm{ml}^{-1}$, and the suspension was incubated at $37^{\circ} \mathrm{C}$ for $2 \mathrm{~h}$ and then at $4{ }^{\circ} \mathrm{C}$ for another $18 \mathrm{~h}$. The cells were removed by centrifugation at $6500 \mathrm{~g}$ for $20 \mathrm{~min}$. This procedure was repeated eight times. The resulting mAbs were used as absorbed mAbs. Mouse ascite induced by myeloma cells was used as a control and designated as P3U1. 
Table 1. Characteristics of $m A$ Abs directed to the flagellin of C. chauvoei

\begin{tabular}{llcc}
\hline \hline mAb & $\begin{array}{c}\text { Passive } \\
\text { protection } \\
\text { test* }\end{array}$ & $\begin{array}{c}\text { H-agglutination } \\
\text { titre }\end{array}$ & $\begin{array}{c}\text { Immobilization } \\
\text { titre }\end{array}$ \\
\hline Mo-41 & Protective & 25600 & 2560 \\
Mo-62 & Protective & 25600 & 2560 \\
Mo-90 & Protective & 25600 & 2560 \\
Mo-114 & Non-protective & $<100$ & $<20$ \\
Mo-2-60 & Non-protective & $<100$ & $<20$ \\
\hline \hline
\end{tabular}

* Mice were injected intraperitoneally with $\mathrm{mAbs}$ and challenged intramuscularly with spores of $C$. chauvoei strain Okinawa (100 m.l.d.) (Tanaka et al., 1987).

$H$-agglutination test and immobilization test. Details of the $\mathrm{H}$-agglutination test and immobilization test were described previously (Tamura \& Tanaka, 1984). Formalin-treated whole cells of $C$. chauvoei strain Okinawa were used as the antigen for the H-agglutination test. The immobilization test was performed as follows. Sterilized tubes containing molten CLB broth (Azechi et al., 1962) with 0.15\% agar were placed in a $50^{\circ} \mathrm{C}$ water bath, and twofold-diluted ascites were added. The cells were grown anaerobically at $37^{\circ} \mathrm{C}$ for $24 \mathrm{~h}$ in heart infusion agar (Difco) supplemented with $5 \%(\mathrm{v} / \mathrm{v})$ defibrinated sheep blood. Single colonies were stab-inoculated into each tube after the soft agar had cooled. The highest dilution of ascites that inhibited the migration of the organism was termed as the immobilization titre of the mAbs.

Immune electron microscopy. C. chauvoei strain Okinawa was cultured anaerobically at $37^{\circ} \mathrm{C}$ and growth was terminated after $16 \mathrm{~h}$ with $0.5 \%$ (v/v) formalin during the late exponential growth phase. Freshly prepared formalin-treated cells were washed gently three times with PBS (g l ${ }^{-1}: \mathrm{NaCl}, 8 \cdot 0 ; \mathrm{KCl}, 0.2 ; \mathrm{Na}_{2} \mathrm{HPO}_{4}, 1 \cdot 15 ; \mathrm{KH}_{2} \mathrm{PO}_{4}, 0.2$; pH 7.2). After centrifugation, the cell pellets were sensitized with $0.2 \mathrm{ml}$ diluted $\mathrm{mAbs}(1: 10$ dilution in PBS containing $1 \%, \mathrm{w} / \mathrm{v}, \mathrm{BSA})$ for $30 \mathrm{~min}$ at $37^{\circ} \mathrm{C}$. The mixture was washed three times with PBS, resuspended in $0.05 \mathrm{ml} \mathrm{1 \%}(\mathrm{v} / \mathrm{v}) \mathrm{BSA}-\mathrm{PBS}$, and adsorbed onto carboncoated grids for $10 \mathrm{~min}$ at room temperature. Excess fluid was removed with a filter paper. The grid was then incubated with anti-mouse IgG and IgM goat serum coupled to $5 \mathrm{~nm}$ gold colloidal particles (1:10 dilution in $1 \%$ BSA-PBS) (E-Y Laboratories) for $30 \mathrm{~min}$ at room temperature. The labelled specimens were negatively stained with $1 \%$ phosphotungstic acid (TAAB Laboratories Equipment) and observed with a JEOL S-100 transmission electron microscope at $80 \mathrm{kV}$.

Purified flagella. Purified flagella were prepared from C. chauveoi strain Okinawa as described previously (Tamura et al., 1984). Briefly, the formalin-treated whole cells were suspended in $0.1 \mathrm{M}$-Tris $/ \mathrm{HCl}$ buffer $(\mathrm{pH} \mathrm{8.0)}$ and the cell suspension was homogenized to shear the flagella with a high-speed homogenizer. Deflagellated cells were removed by centrifugation at $6500 \mathrm{~g}$ for $20 \mathrm{~min}$ and the resulting supernatant was further centrifuged at $77000 \mathrm{~g}$ for $90 \mathrm{~min}$. The pellet was suspended in approximately $20 \mathrm{ml} 1.0 \mathrm{M}$-Tris/HCl buffer and mixed with caesium chloride to a final density of $1.35 \mathrm{~g} \mathrm{~cm}^{-3}$. After centrifugation at $73000 \mathrm{~g}$ for $24 \mathrm{~h}$, a band appearing at $1.33 \mathrm{~g} \mathrm{~cm}^{-3}$ was harvested and dialysed against $0.05 \mathrm{M}-\mathrm{Tris} / \mathrm{HCl}$ buffer $(\mathrm{pH} 7.2)$.

ELISA with absorbed and unabsorbed $m A b s$. ELISA was performed as described previously (Tanaka et al., 1987). Briefly, purified flagella were used as the antigen with a protein content of $0.313 \mu \mathrm{g}$ per well. The wells of micro-ELISA plates were incubated with $100 \mu \mathrm{l} \mathrm{mAbs,} \mathrm{or}$ absorbed $\mathrm{mAbs}$, for $30 \mathrm{~min}$ at room temperature. After washing, the wells were incubated with $100 \mu$ l horseradish peroxidase (HRPO)conjugated anti-mouse IgG (heavy plus light chains) goat serum (1:3000 dilution in PBS containing $5 \%$ foetal calf serum and $0.05 \%$ Tween 20) (Bio-Rad) for $30 \mathrm{~min}$ at room temperature. $O$-Phenylenediamine was used as the substrate, and colour development was terminated with $2 \cdot 0 \mathrm{M}-\mathrm{H}_{2} \mathrm{SO}_{4}$. The $A_{490}$ of each well was observed with a SJeia Auto Reader (Sanko Junyaku).

Competitive binding assay. The competitive binding assay was performed as described previously (Tanaka et al., 1987). Briefly, the mAbs were labelled with HRPO by the method of Nakane \& Kawaoi (1974). The competing antibody was incubated with the antigen for ELISA, as described above, for $4 \mathrm{~h}$ at room temperature, washed six times, and HRPO-labelled mAbs were then added. The percentage competition was determined by the formula $100(A-n) /(A-B)$, where $A$ is the $A_{490}$ in the absence of competing antibody, $B$ is the $A_{490}$ in the presence of homologous antibody and $n$ is the $A_{490}$ in the presence of competitor.

Cyanogen bromide ( $\mathrm{CNBr}$ ) cleavage. $\mathrm{CNBr}$ cleavage of flagellin was performed in $70 \%(\mathrm{v} / \mathrm{v})$ formic acid with an equal amount of $\mathrm{CNBr}$ to purified flagella according to the method of Parish \& Ada (1969). The cleavage mixture was then diluted with distilled water and lyophilized.

Electrophoresis. SDS-PAGE was performed with a Mini-slab gel apparatus (Bio-Rad) by the method of Laemmli (1970). Purified flagella in sample buffer were stacked in polyacrylamide gels and separated in $12 \%$ polyacrylamide at $200 \mathrm{~V}$ for $45 \mathrm{~min}$. A lowmolecular-mass calibration kit (Pharmacia) was used as the molecular mass standard. Gels were stained with Coomassie brilliant blue R-250.

For CNBr-cleaved flagellin, urea-SDS-PAGE was carried out according to the method of Swank \& Munkres (1971). Peptides produced by $\mathrm{CNBr}$ cleavage were separated in $12.5 \%$ polyacrylamide gels containing $8 \mathrm{M}$-urea. Electrophoresis was carried out at $100 \mathrm{~V}$ for $20 \mathrm{~h}$. Fragments of highly purified myoglobin prepared by cleavage with $\mathrm{CNBr}$ were used as molecular mass standards, and peptides were stained with Coomassie brilliant blue R-250.

Immunoblotting assay. Proteins or peptides were electrophoretically transferred to nitrocellulose membranes at $40 \mathrm{~V}$ for $2 \mathrm{~h}$ with a Transblot apparatus (Bio-Rad) by the methanol-Tris/glycine system of Towbin et al. (1979). After being probed with mAbs or anti-Okinawa strain mouse serum, the strips were washed, incubated for $1 \mathrm{~h}$ with HRPO-conjugated anti-mouse IgG goat serum, and washed again. The strips were developed by using HRP colour development reagent (containing 4-chloro-1-naphthol) (Bio-Rad).

\section{Results}

\section{Characteristics of $m A b s$}

Table 1 summarizes the characteristics of the mAbs used. Investigations on the serological reactivity of antiflagella antibodies were carried out with five mAbs reacting with epitopes on flagella of $C$. chauvoei. In $\mathrm{H}$-agglutination and immobilization tests, the mAbs could be divided into two groups. High titre was observed only with the three protective mAbs, each having a similar titre. In contrast, serological reactivity was absent in the two non-protective mAbs. The immobilization titres were approximately 10 -fold lower than the corresponding titres of $\mathrm{H}$-agglutination. 


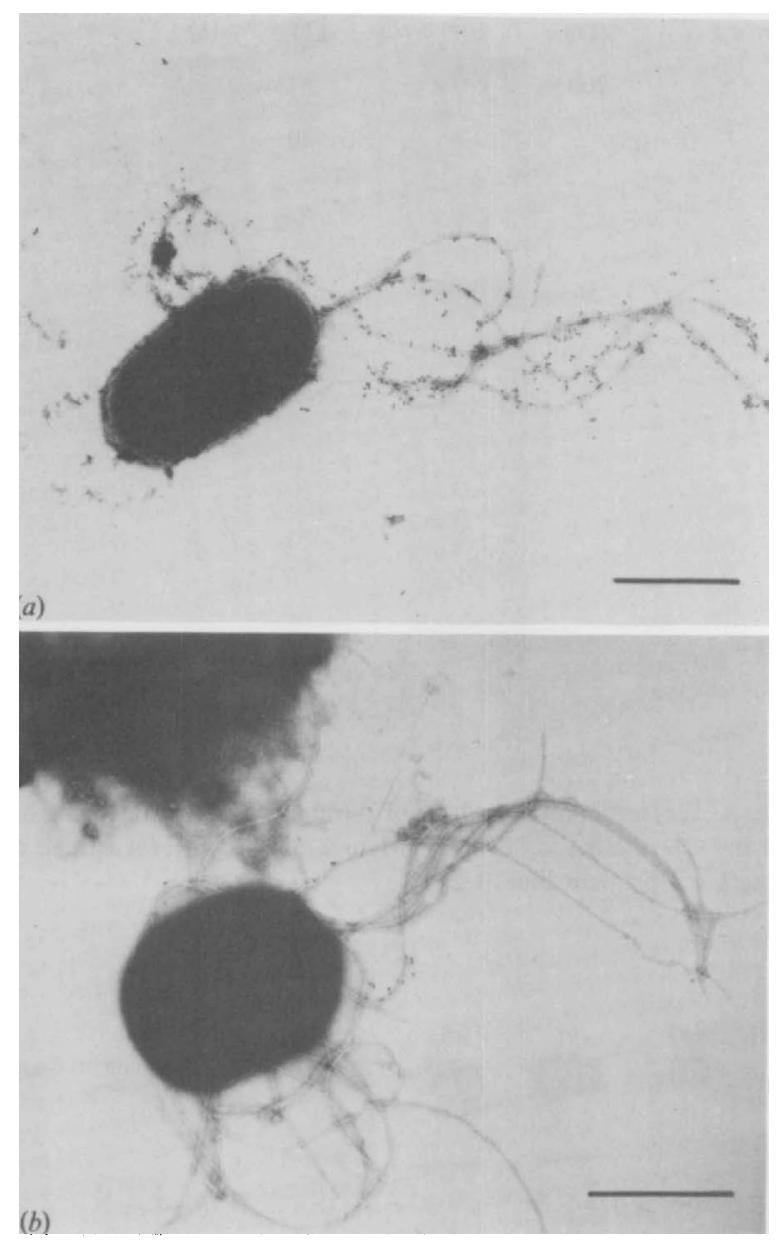

Fig. 1. Immune electron microscopy of $C$. chauvoei strain Okinawa. Whole cells were incubated with mAbs Mo-41 (a) and Mo-114 (b), and processed for immune electron microscopy as described in Methods. Bars, $1 \mu \mathrm{m}$.

\section{Immune electron microscopy}

The location of the epitopes on flagella of formalintreated whole cells was investigated by immune electron microscopy. When mAb Mo-41, which showed a protective effect, was used as the primary antibody, gold particles were observed specifically on the surface of flagellar filaments (Fig. 1 a). mAbs Mo-62 and Mo-90, which also had a protective effect, showed the same behaviour as Mo-41. On the contrary, no noticeable association of gold particles with the flagella was observed in the preparations of Mo-114 (Fig. 1b) and Mo-2-60, which did not show a protective effect, or with P3U1 (control). There were no mAbs that specifically labelled the tips or broken pieces of the flagella.

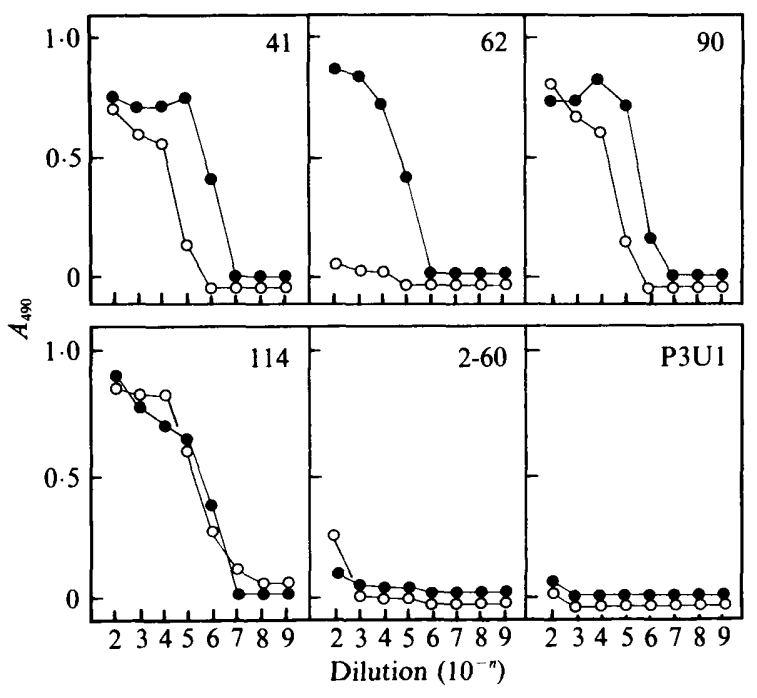

Fig. 2. ELISA reactivity of mAbs directed to the flagellin of $C$. chauvoei. ๑, Unabsorbed mAbs; $O$, mAbs absorbed with formalintreated whole cells of strain Okinawa. The numbers within the boxes indicate mAbs.

\section{ELISA reactivity of $m A b s$}

ELISA was used to confirm the location of epitopes recognized by protective $\mathrm{mAbs}$ on the surface of flagellar filaments. The protective mAbs, Mo-41, Mo-62 and Mo-90, reacted with surface-exposed epitopes on $C$. chauvoei flagella, as shown by absorption with formalintreated whole cells of $C$. chauvoei possessing flagella. This resulted in a substantial reduction of $>0.5$ in maximum $A_{490}$ in ELISA titre (Fig. 2). When mAb Mo-62 was absorbed with whole cells, a much larger reduction in ELISA titre was obtained. In contrast, when the nonprotective mAbs, Mo-114 and Mo-2-60, and P3U1 (control) were absorbed with whole cells, the ELISA titres obtained were similar to unabsorbed mAbs.

\section{Competitive binding assay}

Four mAbs, Mo-41, Mo-62, Mo-90 and Mo-114, were labelled with HRPO and used for a competitive binding assay. Percentage inhibition curves of diluted competitor are shown in Fig. 3. Reciprocal competitions were observed among Mo-41, Mo-62 and Mo-90. These protective mAbs were, however, not blocked with the other two non-protective mAbs. Moreover, non-protective mAb Mo-114 was blocked only by homologous antibody. 


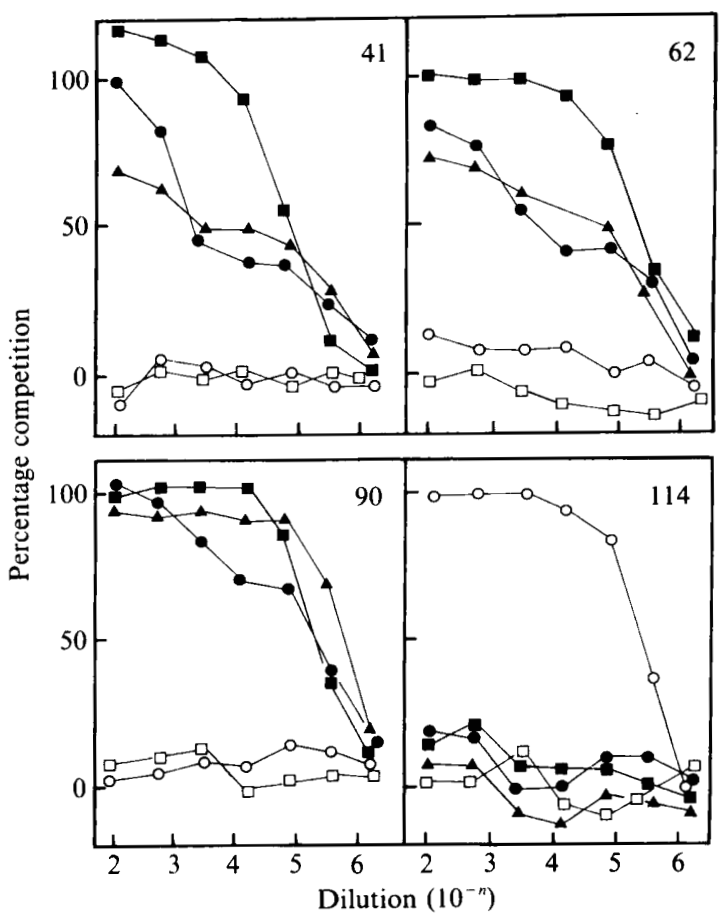

Fig. 3. Competitive inhibition of binding of HRPO-labelled mAbs to flagellin of $C$. chauvoei in the presence of excess concentrations of unlabelled mAbs. $\bullet$, Mo-41; $\boldsymbol{\square}$, Mo-62; $\Delta$, Mo-90; O, Mo-114; $\square$, Mo-2-60. The numbers within the boxes indicated HRPO-labelled mAbs.

\section{Electrophoretic analysis of purified flagella}

The protein profiles of purified flagella were obtained by SDS-PAGE (Fig. 4a). A major protein band with a molecular mass of $56 \mathrm{kDa}$, corresponding to flagellin, and at least two minor protein bands with molecular masses of approximately $200 \mathrm{kDa}$ were found in purified flagella. The location of flagellin epitopes was investigated by $\mathrm{CNBr}$ cleavage of purified flagella. Urea-SDSPAGE of purified flagella cleaved by $\mathrm{CNBr}$ showed major peptides with apparent molecular masses of 33,20 and $6 \mathrm{kDa}$ (Fig. $4 b$ ). There was no cleavage of purified flagella upon incubation in formic acid alone (data not shown).

\section{Immunoblotting assay}

The location of the epitopes recognized by mAbs was investigated by an immunoblotting assay. Fig. 5 shows the typical reaction of $\mathrm{mAbs}$; serum from mice immunized with whole cells of $C$. chauvoei strain Okinawa was used as a control. Protective and non-protective mAbs, Mo-41, Mo-62 and Mo-114, reacted with the $56 \mathrm{kDa}$ protein band, and weakly with a $120 \mathrm{kDa}$ band and two
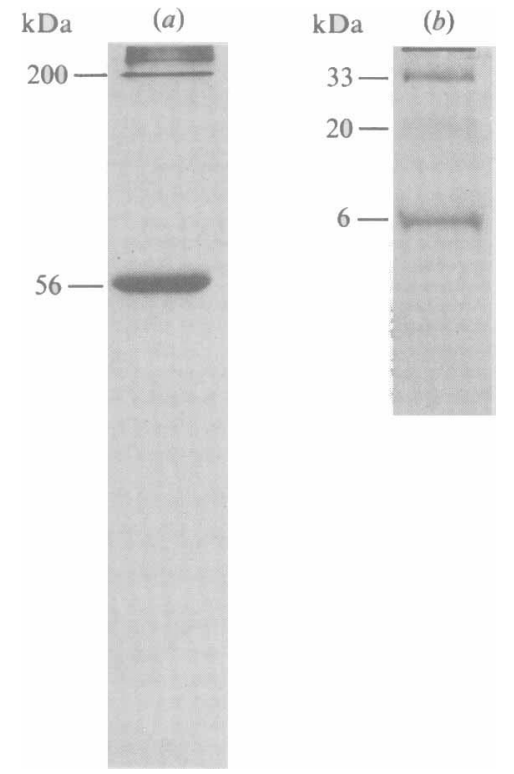

Fig. 4. Electrophoretic analysis of purified flagella with SDS-PAGE $(a)$ and $\mathrm{CNBr}$-cleaved flagellin with urea-SDS-PAGE $(b)$ stained with Coomassie brilliant blue R-250.
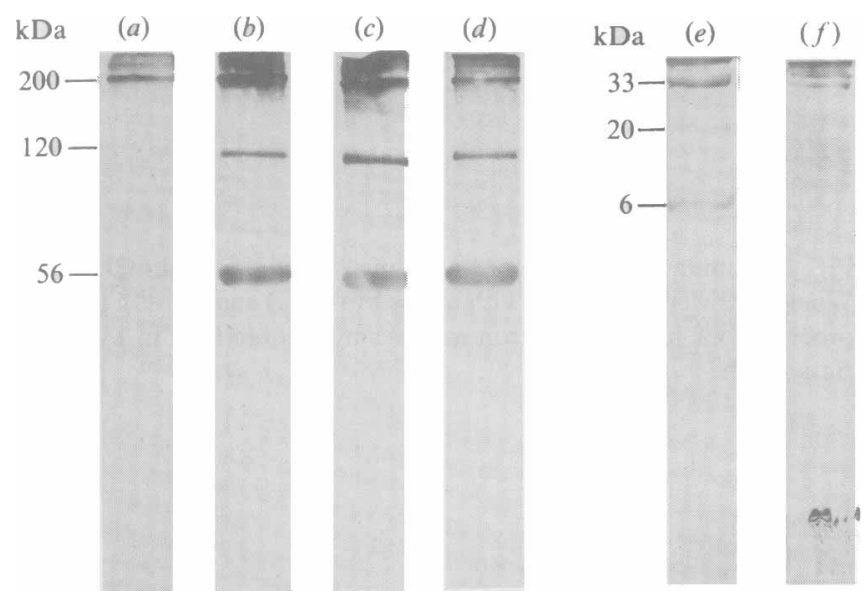

Fig. 5. Immunoblotting analysis of mAbs directed to the flagellin of $C$. chauvoe in SDS-PAGE $(a-d)$ and $\mathrm{CNBr}$-cleaved flagellin in urea-SDSPAGE ( $e$ and $f$ ). (a) Mo-41; (b) Mo-62; $(c$ and $e$ ) Mo-114; $(d$ and $f$ ) mouse anti-strain Okinawa serum.

or more bands of approximately $200 \mathrm{kDa}$ with purified flagella in SDS-PAGE (Fig. $5 a, b, c$ ). mAbs Mo-90 and Mo-2-60 showed a similar reaction pattern although the reactions were weak (data not shown). Anti-Okinawa strain serum, in comparison with the mAbs, also showed the same reaction pattern. The reaction of mAbs with $\mathrm{CNBr}$-cleaved flagellin was also examined. Only Mo-114 reacted with three peptides of $\mathrm{CNBr}$-cleaved flagellin 
(Fig. $5 e$ ). In contrast, the other mAbs result in a loss of the immunoblotting reaction (data not shown). With $\mathrm{CNBr}$-cleaved flagellin in urea-SDS-PAGE, however, anti-Okinawa strain serum reacted solely with a fragment of $33 \mathrm{kDa}$.

\section{Discussion}

Flagella of $C$. chauvoei are organelles which help to induce immune resistance mechanisms, as demonstrated by the mouse protection test (Tamura et al., 1984). Flagellar antibodies appear to exert their protective effect by opsonic activity such that opsonized $C$. chauvoei are eliminated by polymorphonuclear leucocytes (Tamura \& Tanaka, 1984, 1987). Flagella have, therefore, been considered as one of the candidates for vaccines against blackleg in cattle and sheep. Our previous study showed that three mAbs directed to the flagellin of C. chauvoei, Mo-41, Mo-62 and Mo-90, had protective effects in mice (Tanaka et al., 1987). The present study was aimed at elucidation of the protective antigen on the flagella of $C$. chauvoei using these $\mathrm{mAbs}$.

Immunogold-labelled $\mathrm{mAbs}$ have been used to visualize epitopes on the flagella of $C$. chauvoei. Protective mAbs specifically labelled the surface of the flagellar filament, while non-protective mAbs did not. These results indicate that the epitopes recognized by the protective $\mathrm{mAbs}$ are surface-exposed on the flagellar filament of $C$. chauvoei, and those of non-protective mAbs are not surface-exposed; this could be explained by an inaccessibility of non-protective mAbs to their epitopes. Inadequate exposure of non-protective epitopes on the surface of the flagellar filament is confirmed by the absence of $\mathrm{H}$-agglutination and immobilization activity with non-protective mAbs. Similar phenomena have been reported in Campylobacter jejuni with the mAbs directed to the internal epitopes on flagella (Newell, 1986).

These findings were also confirmed by absorption tests of ELISA reactivity. After absorption with whole cells of C. chauvoei strain Okinawa possessing flagella, ELISA reactivities of the protective $\mathrm{mAbs}$ were significantly reduced compared with those of the unabsorbed mAbs, indicating that protective epitope was exposed on the surface of the flagellar filament.

To ascertain whether these three protective mAbs reacted with the same epitope, a competitive binding assay was performed. When protective mAbs were conjugated to HRPO, competition was observed only among protective mAbs, indicating that their epitopes are identical or topographically overlapping. In contrast, non-protective epitopes are obviously in a position separate from the protective epitope, and each epitope of the non-protective mAbs is topographically separated from remaining epitopes. On the basis of these results, we have defined three distinguishable epitopes on the flagellin of $C$. chauvoei.

The epitopes participating in protection, when coupled with our previous observations (Tanaka et al. 1987), were species-specific and surface-exposed on the flagellar filament. Interestingly, however, the epitopes of the non-protective mAbs were cross-reactive and not surface-exposed implying that, in this case, the nonprotective epitopes may carry conserved primary sequences among various clostridia. ELISA reactivity with non-protective mAbs also suggested that the conserved sequence was internally located in the flagellar filament. Logan \& Trust (1986) reported that the flagella of $C$. jejuni have both internal and surface-exposed epitopes, and suggest that the immunodominant surfaceexposed epitopes are serospecific, and much of the conserved sequence is internally located in the native flagellar filament. Epitopes in polymeric flagellin also play a major role in the serospecificity of Salmonella flagella (Ibrahim et al., 1985).

In immunoblotting analysis of purified flagella, all five mAbs reacted with several proteins, showing one clear band of $56 \mathrm{kDa}$, which corresponded to the flagellin monomer, and at least three weak bands ranging from $120-200 \mathrm{kDa}$, which are polymeric forms of the flagellin monomer, as described previously (Tanaka et al., 1987). Subsequently, the location of epitopes recognized by the $\mathrm{mAbs}$ was investigated using $\mathrm{CNBr}$-cleaved flagellin. Coomassie brilliant blue-stained urea-SDS-PAGE gels of $C$. chauvoei flagellin cleaved by $\mathrm{CNBr}$ displayed major peptides with apparent molecular masses of 33, 20 and $6 \mathrm{kDa}$. The most important result in this study was the observation that the protective mAbs did not react with $\mathrm{CNBr}$-cleaved peptides of flagellin in the immunoblotting analysis, while the non-protective $\mathrm{mAb}$, Mo-114, did. These results suggest that protective mAbs may recognize conformational epitopes derived from the secondary structure of flagellin. On the other hand, the non-protective Mo-114 reacted with three peptides of flagellin cleaved by $\mathrm{CNBr}$, indicating that at least one non-protective mAb may recognize a linear, and conformation-independent epitope in the flagellin. As yet we are unable to define the protective or nonprotective epitopes on the flagellin molecule. The identification of protective conformational epitopes in the flagellin of $C$. chauvoei has prompted us to consider the importance of such a determinant as a component in the development of a vaccine that would be effective against blackleg disease. Further studies on the location of protective epitopes that involve the use of these antiflagellar $\mathrm{mAbs}$ are necessary in order to elucidate the native form of the protective antigen on the flagella of $C$. chauvoei. 


\section{References}

AtTridge, S. R. \& Rowley, D. (1983). The role of the flagellum in the adherence of Vibrio cholerae. Journal of Infectious Diseases 147, 864-872.

Azechi, H., Seto, K., Mukawa, Y. \& Suzuki, K. (1962). Studies on the potency test of blackleg vaccine. Japanese Journal of Veterinary Sciences 24, 267-275.

Carsiotis, M., Weinstein, D. L., Karch, H., Holder, I. A. \& O'BRIEN, A. D. (1984). Flagella of Salmonella typhimurium are a virulence factor in infected $\mathrm{C} 57 \mathrm{BL} / 6 \mathrm{~J}$ mice. Infection and Immunity 46, 814-818.

IbraHiM, G. F., Fleet, G. T., Lyons, M. J. \& WALkeR, R. A. (1985). Immunological relationships between Salmonella flagellins and between these and flagellins from other species of Enterobacteriaceae. Medical Microbiology and Immunology 174, 101-113.

LAEMMLI, U. K. (1970). Cleavage of structural proteins during the assembly of the head of bacteriophage T4. Nature, London 227, 680-685.

Logan, S. M. \& Trust, T. J. (1986). Location of epitopes on Campylobacter jejuni flagella. Journal of Bacteriology 168, 739-745.

Montie, T. C., Doyle-Huntzinger, D. Craven, R C. \& Holder, I. A. (1982). Loss of virulence associated with absence of flagellum in an isogenic mutant of Pseudomonas aeruginosa in the burned-mouse model. Infection and Immunity 38, 1296-1298.

Montie, T. C., Drake, D., Sellin, H., Slater, O. \& Edmonds, S. (1987). Motility, virulence, and protection with a flagella vaccine against Pseudomonas aeruginosa infection. Antibiotics and Chemotherapy 39, 233-248.

MorookA, T., UMEdA, A. \& Amako, K. (1985). Motility as an intestinal colonization factor for Campylobacter jejuni. Journal of General Microbiology 131, 1973-1980.

Nakane, P. K. \& KaWaOI, A. (1974). Peroxidase-labelled antibody: a new method of conjugation. Journal of Histochemistry and Cytochemistry 22, 1084-1091.

NEWELL, D. G. (1986). Monoclonal antibodies directed against the flagella of Campylobacter jejuni: production, characterization and lack of effect on the colonization of infant mice. Journal of Hygiene 96, 131-141.

NeWELl, D. G., MCBRIDE, H. \& DOLBY, J. M. (1985). Investigation on the role of flagella in the colonization of infant mice with Campylobacter jejuni and attachment of Campylobacter jejuni to human epithelial cell lines. Journal of Hygiene 95, 217-227.

PARISH, C. R. \& ADA, G. L. (1969). Cleavage of bacterial flagellin with cyanogen bromide. Biochemical Journal 113, 489-499.

Resnick, I. G., Ford, C. W., Shackleford, G. M. \& Berry, L. J. (1980). Improved protection against cholera in adult rabbits with a combined flagellar-toxoid vaccine. Infection and Immunity 30, 375-380.

SwaNk, R. T. \& MuNKRES, K. D. (1971). Molecular weight analysis of oligopeptide by electrophoresis in polyacrylamide gel with sodium dodecyl sulfate. Analytical Biochemistry 39, 462-477.

TAMURA, Y. \& TANAKA, S. (1984). Effect of antiflagellar serum in the protection of mice against Clostridium chauvoei. Infection and Immunity 43, 612-616.

TAMURA, Y. \& TANaKA, M. (1987). Opsonic activity of antiflagellar serum against Clostridium chauvoei by mouse polymorphonuclear leucocytes. Veterinary Microbiology 14, 81-86.

Tamura, Y., Minamoto, N. \& Tanaka, S. (1984). Demonstration of protective antigen carried by flagella of Clostridium chauvoei. Microbiology and Immunology 28, 1325-1332.

Tanaka, M., Hirayama, N. \& Tamura, Y. (1987). Production, characterization, and protective effect of monoclonal antibodies to Clostridium chauvoei. Infection and Immunity 55, 1779-1783.

Towbin, H., StaEhelin, T. \& Gordon, J. (1979). Electrophoretic transfer of proteins from polyacrylamide gels to nitrocellulose sheets: procedure and some applications. Proceedings of the National Academy of Sciences of the United States of America 76, 4350-4354.

Weinstein, D. L., Carsiotis, M., Lissner, C. R. \& O'Brien, A. D. (1984). Flagella help Salmonella typhimurium survive within murine macrophages. Infection and Immunity 46, 819-825.

YANCEY, R. J., WILLIS, D. L. \& BERRY, L. J. (1979). Flagella-induced immunity against experimental cholera in adult rabbits. Infection and Immunity 25, 220-228. 